
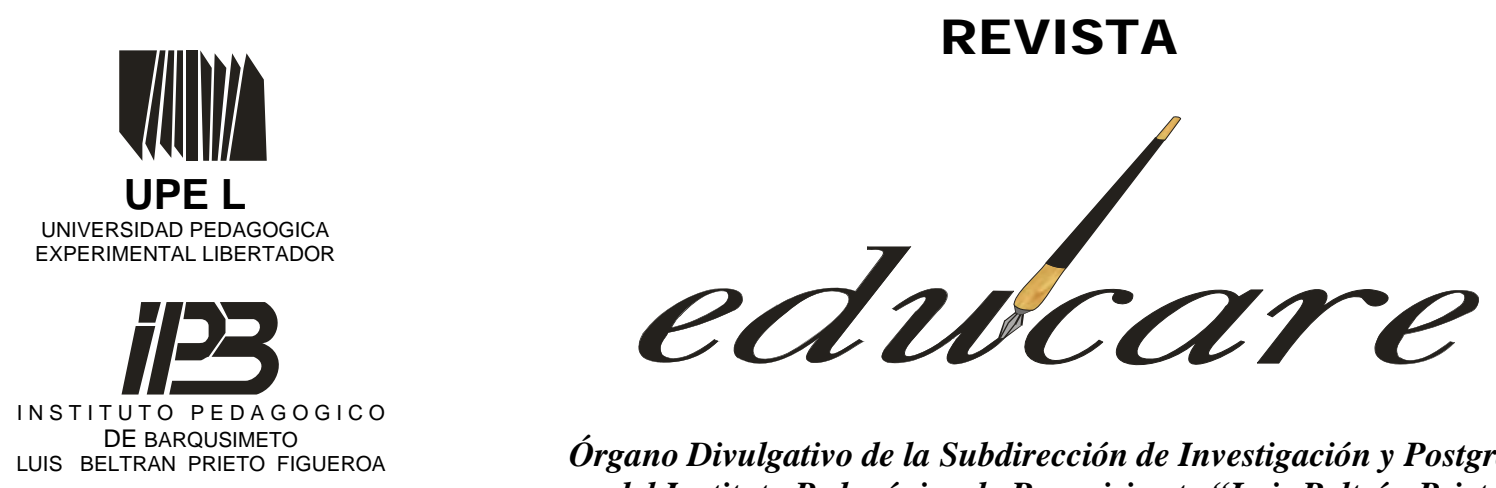

Órgano Divulgativo de la Subdirección de Investigación y Postgrado del Instituto Pedagógico de Barquisimeto "Luis Beltrán Prieto

Figueroa"

BARQUISIMETO - EDO. LARA - VENEZUELA

NUEVA ETAPA

VERSION ELECTRÒNICA

DEPOSITO LEGAL: ppi201002LA3674

Volumen $14 \mathrm{~N}^{\circ} 2$

ISSN: 2244-7296

Mayo-Agosto 2010

\title{
PENSAMIENTO MATEMÁTICO CREATIVO EN LA EDUCACIÓN POLITECNICA A TRAVÉS DE NIVELES DE LA ACTIVIDAD MATEMÁTICA
}

CREATIVE MATHEMATICAL THINKING THROUGH LEVELS OF MATHEMATICAL ACTIVITY AT ENGINEERING UNIVERSITES

\section{Pablo Adolfo Pérez Giuvuva}

\section{Universidad Nacional Experimental Politécnica Antonio José de Sucre Vicerrectorado Barquisimeto}




\section{PENSAMIENTO MATEMÁTICO CREATIVO EN LA EDUCACIÓN POLITECNICA A TRAVÉS DE NIVELES DE LA ACTIVIDAD MATEMÁTICA.}

\section{CREATIVE MATHEMATICAL THINKING THROUGH LEVELS OF MATHEMATICAL ACTIVITY AT ENGINEERING UNIVERSITES}

$\underline{\text { ENSAYO }}$

Recibido:12-03-10

\author{
Pablo Adolfo Pérez Givuva* \\ UNEXPO \\ Vicerrectorado Barquisimeto
}

\begin{abstract}
RESUMEN
El propósito del presente ensayo de naturaleza argumentativa, es establecer que la creatividad matemática en la educación politécnica se manifiesta en la transición del primer al segundo nivel, de los tres que constituyen la actividad matemática; mediante el desarrollo de estrategias didácticas que rebasen el carácter instrumental de la matemática para ingenieros, que corresponde a ese primer nivel. Este enfoque, fundamentado en las ideas de Changeux y Connes (1993), es más adecuado que el del modelo clásico del pensamiento matemático creativo según PoincaréHadamard, constituido por cuatro fases, cuya implementación presenta serias dificultades relativas a tiempo, espacio y carácter imprevisto e incontrolado de las fases intermedias. Se concluye que superar el primer nivel supone ejecutar actividades didácticas que trasciendan la simple aplicación de algoritmos y busquen cuestionarlos, mejorarlos o reconstruirlos, crear modelos y desarrollar proyectos; así como involucrarse emocionalmente en el aprendizaje.
\end{abstract}

Descriptores: Pensamiento matemático creativo, niveles de actividad matemática y matemática aplicada a la ingeniería.
Aceptado: 28-07-10

\begin{abstract}
This essay aims to establishment that mathematical creativeness at engineering education is shown during the transition from the first to the second level of mathematical activity. It is possible thanks to the development of didactic strategies that overcome the instrumental character of the subject. This approach, supported on Changeux and Connes' ideas, is believed to be more appropriate than Poincaré's classical model of creative mathematical, which application presents serious difficulties related to time, space, and the uncontrollable and unforeseeable character of intermediate stages. It is concluded that to overcome the first level, didactic activities that go beyond the mere application of algorisms, and projects development are needed, as well as getting emotionally involved in the learning process.
\end{abstract}

Keywords: Creative mathematical thinking, levels of mathematical activity, engineering applied mathematics

\section{INTRODUCCIÓN}

\footnotetext{
* Pablo Adolfo Pérez Givuva .Profesor de Ciencias Experimentales, Mención Física, egresado de la U.P.E.L, Venezuela, Magíster en Matemática Aplicable, egresado del Convenio MIM (UCLA-UNEXPO-UPEL). Adscrito al Departamento de Estudios Generales y Básicos de la UNEXPO-Barquisimeto, Sección de Matemáticas. Estudiante del programa doctoral PIDE, Convenio UCLA-UNEXPO-UPEL. Línea de Investigación: Creatividad. papgivuva@yahoo.es
}

Revista EDUCARE, Volumen 14, Número 2, Mayo-Agosto 2010 . ISSN: 2244-7296

Página 100 
Cuando nos interesamos por el estudio del pensamiento matemático creativo, conocemos que los primeros aportes a sus bases teóricas son las ideas de Poincaré (1963), quien establece los fundamentos básicos de lo que denominaremos Modelo Clásico de Creatividad Matemática, y luego están las contribuciones hechas, entre otros, por Hadamard (1996), Changeux y Connes (1993) y más recientemente, Penrose (1996). Este modelo entraña cuatro fases: la preparación, la incubación, la iluminación y la verificación; y ha sido deducido a partir de las experiencias creativas de científicos matemáticos profesionales, de prestigio mundial y para los que la creación matemática constituye la ocupación esencial.

La manifestación práctica del modelo, cuyo producto máximo sería la creación o el descubrimiento de un nuevo conocimiento matemático, no está limitada por el tiempo que puede consumir una investigación ni los espacios disponibles por el investigador en su actividad creadora. La ausencia de estas restricciones lo hace muy apropiado para las fases intermedias del modelo: incubación e iluminación, caracterizadas por ser el efecto de actividades del inconsciente y que pueden manifestarse en lugares y momentos impredecibles, escapando así al control de la parte consciente de la mente. Por estas razones consideramos que tales experiencias son difíciles de reproducir en un estudiante o profesor de una escuela de ingeniería, limitados por un semestre de clases y confinados a las cuatro paredes de un aula, y donde el objetivo no es formar matemáticos profesionales sino ingenieros, cuyos modelos de conducta son diferentes.

Por lo tanto, consideramos que las bases para el pensamiento matemático creativo en una escuela de ingeniería, con las naturales restricciones de tiempo, espacio y objetivos de formación profesional, deben elaborarse a partir de un estudio crítico de los aportes del modelo clásico y los criterios expuestos por matemáticos cuando reflexionan sobre su proceso creativo. Proponemos considerar las ideas expuestas en Changeux y Connes (Ob. Cit.) como punto de referencia inicial para la reflexión y crítica, tratando de aportar un cuerpo de ideas adecuado y factible, donde puedan deducirse lineamientos para proponer y realizar actividades didácticas en matemáticas que fomenten la creatividad en un aula de clases de ingeniería y en un semestre.

Nuestra tesis es que la condición para impulsar la creatividad en matemáticas en la educación politécnica es proponer y ejecutar las estrategias docentes que originen una 
transición del primer al segundo nivel de la actividad matemática, según las ideas expuestas por los autores antes citados. Para fundamentar esta tesis, comenzaremos por una consideración crítica del modelo clásico de creatividad matemática, la definición de creatividad asociada y sus dificultades para ser aplicado en una clase y en un semestre, luego discutiremos el modelo propuesto, la definición de creatividad asociada y los criterios que utiliza para delimitar los niveles de la actividad matemática, argumentando que el lugar que ocupa en esos niveles la matemática para ingeniería origina limitaciones al desarrollo de la creatividad y posteriormente sugerimos las acciones didácticas que deberían tomarse para fomentarla.

Hemos evitado a lo largo del artículo la discusión entre los términos descubrir e inventar, porque en la matemática ella conduce a polémicas relacionadas con la filosofía y los fundamentos de esta ciencia. En efecto, para el platonismo la matemática está constituida por entes ideales y sus relaciones, que existen fuera e independientes del ser humano. En esta actitud filosófica, el investigador "no puede inventar nada porque todo ya está allí. Todo lo que puede hacer es descubrir” (Davis y Hersh, 1998, p. 318). Mientras que el constructivismo es opuesto al platonismo. Esta actitud filosófica considera como matemática verdadera sólo la que puede ser obtenida mediante una construcción finita. Por consiguiente, para ella el investigador inventa la matemática, no la descubre. El formalismo es la tercera actitud filosófica que considera a la matemática cadenas de símbolos que sólo adquieren significado cuando entrañan una particular interpretación física. En razón del propósito del artículo y los límites de su extensión no trataremos estas ideas.

\section{EL MODELO CLÁSICO DE CREATIVIDAD MATEMÁTICA}

El modelo clásico del pensamiento matemático creativo establece sus fundamentos en las ideas expuestas por Poincaré (1963). El núcleo de este modelo son las cuatro fases indicadas anteriormente, dentro de un marco general de actitudes y comportamientos asociados al investigador matemático. Veamos los dos aspectos de este marco general y luego las fases.

El primer aspecto es lo que Poincaré denomina el sentido del orden matemático, entendiendo por tal la intuición de poder percibir el orden subyacente a todo argumento 
matemático más o menos extenso cuyo fin es demostrar alguna proposición matemática, conocida como Teorema en nuestro vocabulario particular. Un razonamiento matemático no es una simple yuxtaposición de sus elementos constituyentes, llamados silogismos; esos silogismos están colocados en un cierto orden, y ese orden es mucho más importante que la relevancia particular de cada uno de ellos. Quien aspire a ser matemático debe esforzarse por desarrollar este sentido matemático, sólo así podrá percibir el orden que posibilita comprender rápidamente el conjunto del razonamiento, de tal modo que si olvida uno de los elementos constituyentes, sería un mal menor pues cada uno vendrá a colocarse en el cuadro preparado por el matemático, sin mayor esfuerzo de la memoria.

A partir de este planteamiento, Poincaré indica que no todas las personas pueden llegar a ser matemáticos creadores, del mismo modo que no todos llegarán a ser pintores o músicos innovadores. "se concibe que este sentimiento, esta intuición del orden matemático que nos hace adivinar las armonías y relaciones ocultas, no puede pertenecer a todo el mundo” (Poincaré, 1963, p. 42). Clasifica a las personas de acuerdo al grado de desarrollo de este sentido matemático y de su capacidad de memoria. En el estrato inferior están los que no poseen ese sentido y tienen una memoria y capacidad de atención más bien mediocre. Serán incapaces de comprender las matemáticas un poco elevadas, siendo este el caso de la mayoría de las personas. En el estrato intermedio están los que tienen ese sentido en pequeño grado pero una buena memoria y gran capacidad de atención; pueden aprender de memoria los detalles, el orden presentado; comprenden las matemáticas y a veces las aplican pero serán incapaces de crear. Y en el estrato superior, están quienes tienen en grado elevado la intuición de ese sentido matemático y entonces comprenden las matemáticas, aunque su memoria no sea notable; pueden llegar a ser creadores y tratan de inventar con más o menos éxito, según hayan desarrollado esa intuición. Debemos indicar que esta clasificación, que establece límites a la creatividad, hace referencia al matemático puro profesional.

El segundo aspecto del marco general es el sentimiento estético mediante el cual el matemático atribuye belleza y elegancia a ciertos entes matemáticos. Poseer y desarrollar una especie de emoción estética es una condición básica de la actitud creativa en matemáticas. “Podemos sorprendernos de ver invocar la sensibilidad con motivo de demostraciones matemáticas que aparentemente no podrían interesar más que a la inteligencia. Esto sería olvidar el sentimiento de la belleza matemática, de la armonía de los números y de las formas, 
de la elegancia geométrica. Es un auténtico sentido estético que todos los verdaderos matemáticos conocen” (Poincaré, ob.cit., p. 48). Los entes matemáticos a los que se les atribuye belleza y elegancia, que suscitan la emoción estética en el investigador, son aquellos cuyos elementos están armoniosamente dispuestos, de manera que la mente sensible puede, sin esfuerzo aparente, abarcar todo el conjunto y penetrar en los detalles. Este criterio estético es crucial en la invención matemática.

La definición de creatividad asociada a este modelo la enuncia Poincaré (ob.cit., p. 42):”Inventar es discernir, es elegir”, pero esta elección la realiza el inconsciente del investigador y utiliza un criterio estético. Puede ocurrir en el momento y en el sitio más inesperado. No se puede asociar ni con un aula de clases ni con un lapso académico determinado y escapan a nuestro control consciente. "Entre las numerosas combinaciones que el yo subconsciente ciegamente ha formado, casi todas carecen de interés y de utilidad, pero por eso mismo no excitan la sensibilidad estética; la consciencia no las conocerá jamás; algunas solamente son armoniosas y por consiguiente a la vez inútiles y bellas, serán capaces de conmover la sensibilidad especial del geómetra (...) y que una vez excitada llamará sobre ella nuestra atención y le dará así ocasión de volverse consciente” (ibidem, p. 49). Esta iluminación puede engañar al matemático pero entonces no resiste la cuarta fase de verificación. Hay una bella metáfora para representar estas ideas: el inconsciente del matemático esta provisto de una criba delicada, que sólo deja pasar las combinaciones bellas, armoniosas y útiles hacia el yo consciente e indica que quien este desprovisto de ella "no será jamás un verdadero inventor” (ibdem., p. 49).

Lo expuesto se puede resumir con las siguientes palabras: "Es claro que ningún descubrimiento o invención significativo puede ocurrir sin la voluntad de hallar. Pero con Poincaré, vemos algo más, la intervención del sentido de la belleza juega su parte como un indispensable medio para hallar. Hemos alcanzado una doble conclusión: la invención es elección y esta elección esta imperativamente gobernada por el sentido de la belleza científica.” (Hadamard, 1996, p. 31). El sentido del orden y el sentido estético son los dos aspectos del marco general del modelo clásico de creatividad, y ambos están referidos a la práctica diaria de los investigadores matemáticos profesionales para los que la matemática es su modo de vida. En nuestra opinión, pretender inculcar estos altísimos niveles de desempeño en estudiantes universitarios de carreras técnicas entraña dificultades prácticas. 
Las fases del modelo clásico del pensamiento matemático creativo son: la preparación; que corresponde a una etapa de estudio consciente y arduo de algún problema de cierta complejidad, donde el investigador concentra su atención durante un tiempo prolongado. La incubación; donde la preparación consciente pone en marcha un mecanismo inconsciente que busca la solución y evalúa las combinaciones resultantes sobre la base de un criterio estético. La iluminación; donde la combinación que satisface el criterio estético del inconsciente súbitamente emerge a la consciencia y, por último, la fase de verificación; donde se lleva a cabo un posterior trabajo consciente para verificar la iluminación, formularla de manera precisa y, tal vez, seguir sus consecuencias.

Esta teoría, de enorme influencia entre matemáticos, psicólogos y educadores matemáticos ha sido atribuida a psicólogos y también a matemáticos. Aceptamos como veredicto definitivo lo expuesto por Hadamard (ob.cit).en el prólogo de su libro: "Este estudio, como todo lo que puede ser escrito sobre invención matemática, primero fue inspirado por la famosa conferencia de Henry Poincaré ante la sociedad de psicólogos de Paris...” (Hadamard, ob.cit., p xv). Un rasgo notable de uno de los mejores matemáticos de todos los tiempos fue el ocupar parte de su atención a la creatividad y el aprendizaje en las matemáticas.

En una investigación cualitativa, Sriraman (2004) verificó en un grupo de investigadores matemáticos las cuatro fases del proceso creativo según las ideas de Poincaré y Hadamard. La preparacón consistió de varios enfoques para establecer las bases teóricas del problema matemático investigado, y comprendierón leer la literatura existente, dialogar con otros colegas sobre el tema específico y ensayar diversos métodos de resolución aprovechando el aprendizaje de anteriores experiencias. Los matemáticos entrevistados investigaban varios problemas simultáneamente previendo que si en un tiempo determinado no se percibian avances de solución en uno de ellos, cambiaban a otro. Todos considerarón esta fase como la más importante y dificil del proceso creativo.

Al prolongado estudio anterior le siguió la fase de incubación, donde el problema específico era dejado de lado y la preparación se repetía para otro de los problemas a cargo del matemático. Esta estrategía es romper el hilo del pensamiento en relación al primer problema, y origina en la mente una transición de un trabajo conciente a otro inconciente. Los matemáticos afirmarón que es el momento en el cual el problema comienza a hablarle al 
investigador y creen que el lado intuitivo del cerebro comenzaba a comunicarse con el lógico, conjeturando que ello no es posible en el nivel conciente de la preparación.

En relación a la iluminación manifestarón es imprevista. Unos reportarón el avance cuando iban a dormir o al caminar, otros al hablar con alguien acerca del problema. Afirman que la incubación es el momento en el cual la oportunidad toca a la puerta, pero hay que estar preparado para acceder al llamado.

Finalmente se verifica la idea iluminadora mediante la construcción de la demostración matemática. Sriraman (ob.cit.) halló que los matemáticos buscaban coherencia de sus resultados con otros diferentes ya demostrados en el área de estudio. Si lo es y además se corresponde a la estructura del área, se ocupan de construir la demostración formal. El punto final del proceso creativo es la publicación en una revista científica reconocida, luego de ser aprobados sus resultados por el grupo de expertos del área.

En nuestra opinión, tomar como referencia un modelo que surgió de la reflexión de matemáticos insignes sobre su práctica, para fomentar la creatividad en un entorno universitario entraña serias dificultades; sobre todo en cuanto a la ejecución de las fases intermedias de incubación e iluminación. Ellas son realizadas por el inconsciente, sobre el que no tenemos pleno control consciente. El consciente prepara las condiciones para la invención en la primera fase y verifica, formaliza y contextualiza los hallazgos en la última. No podemos dirigir la actividad del inconsciente; más aún, según teorías psicológicas contemporáneas, es el inconsciente quien controla al consciente.

Consideremos con mayor detalle estas dificultades. El desarrollo del proceso de enseñanza y aprendizaje de la matemática en una facultad de ingeniería ocurre en un semestre y en un aula, lo que pone límites en tiempo y espacio a lo que hay que aprender y cómo demostrar ese aprendizaje. Las fases intermedias, de incubación y de iluminación, ejecutadas por el inconsciente no admiten límites de esta clase. Por ejemplo, una sugerencia habitual entre los investigadores en aras de propiciar la iluminación, consiste en abandonar, durante un intervalo, un problema que ha consumido mucho tiempo y agotado los recursos que se disponían para su solución; para reanudarlo un tiempo después. Se busca con ello relajar la concentración y facilitar el pensamiento lateral: "Yo, personalmente, lo hago en muchos casos y siempre lo recomendé a los principiantes que me consultaron.” (Hadamard, ob.cit.; p. 9). Evidentemente la aplicación de esta sugerencia está muy bien para un investigador 
matemático pero para un estudiante de ingeniería en un examen le ocasionaría ser aplazado, porque la suspensión del hilo del pensamiento le ocasionaría perder minutos preciosos. En una evaluación corriente, la concentración mental debe ser intensa y continua, lo que se corresponde con el segundo nivel, donde no se rompe el hilo del pensamiento.

Las fases de preparación, incubación, iluminación y verificación son las fases del proceso creativo en matemática propuestas por Poincaré (ob.cit.) y aceptadas por muchos matemáticos profesionales. Esta afirmación no niega ni excluye el hecho de que estas fases se presenten también a lo largo de todo proceso de enseñanza y aprendizaje, puesto que el estudiante no aprende únicamente en el salón de clases sino que todos sus momentos de estudio son aprendizajes y en ellos se suceden también la suspensión momentánea del hilo del pensamiento.

Siguiendo con este orden de ideas, la fase de iluminación puede ocurrir en lugares y momentos imprevistos y no necesariamente en un aula de clases o en un semestre académico. Según relatos que hemos leído, ha ocurrido en el momento del despertar, al subir a un tranvía, al cruzar una calle junto a un amigo... ¿cómo esperar que la inspiración nos llegue cuando más la necesitamos y en el lugar que estamos? Eso lo decidirá nuestro inconsciente, después de examinar las combinaciones que ha ido formando, tomando como base los conocimientos aportados por la fase de preparación.

Ante esta situación, debemos discutir y reflexionar sobre las condiciones que propician la creatividad matemática bajo las limitaciones inherentes a una formación universitaria regida por planes de estudio invariables y donde la enseñanza y el aprendizaje deben ser evaluados en periodos de tiempos definidos. Ello implicaría considerar las ideas del modelo tradicional de creatividad matemática de una manera crítica y complementarlas con los aportes de otros científicos en el campo, así como de educadores que hayan estudiado el tema.

\section{MODELO DE CREATIVIDAD PROPUESTO SEGÚN LAS IDEAS DE CHANGEUX Y CONNES}

Las dificultades indicadas para la aplicación práctica del modelo clásico de creatividad matemática en un aula y un semestre, nos conducen a examinar un posible modelo de creatividad basado en la clasificación de la actividad matemática en tres niveles. El ascenso de un nivel al otro surge de las características de las actividades de aprendizaje de cada uno, las 
cuales exigen un grado cada vez mayor de actitudes creativas. Las ideas de este posible modelo surgieron de la discusión entre el neurobiólogo Changeux (ob.cit.) y el matemático medalla Connes (ob.cit). Veamos estos niveles y el lugar que ocupa la matemática para ingenieros en ellos.

\section{El primer nivel}

El primer nivel se define por la facultad de calcular y de saber aplicar bien y rápidamente una receta o algoritmo dado. Las operaciones a ejecutar pueden ser muy complicadas, sin embargo, sin importar su grado de complejidad, esta receta o algoritmo siempre se conoce previamente aunque no se comprenda en absoluto. Un ejemplo de Calculo IV podría ser aplicar el método de separación de variables para resolver la ecuación diferencial parcial de la cuerda vibrante, cuyo método se conoce desde hace mucho tiempo. No es posible ninguna variación ni cambio de estrategia para resolver el problema. Este es el nivel de un saber instrumental, donde la matemática es considerada una ciencia auxiliar a la ingeniería, de modo análogo a como se considera la biología en medicina o el inglés en una carrera técnica; y se corresponde con el de las computadoras actuales.

Otro ejemplo de matemática para ingenieros es el de resolver un sistema de ecuaciones diferenciales mediante la transformada de Laplace, de nivel de complejidad Matemáticas Especiales, cuarto semestre de ingeniería. Ya existen los mecanismos preestablecidos que permiten dar una respuesta precisa. Es un procedimiento de naturaleza algorítmica y numérica. Realizar estas operaciones no tiene consecuencias prácticas sobre la manera como se llevan a cabo. “A menos que pasemos a otro nivel, el método no cambia, se aplica sin entender por qué...Por automatismo.” (Changeux y Connes, 1993, p. 86). No obstante, los algoritmos y el saber instrumental, aunque no sean más que recetas, tienen sus beneficios: al aplicarlos se obtienen resultados mucho más rápidamente que los que haría el cerebro si los desconociese. Además la matemática al formalizarse para ser enseñada y aplicada asume la forma de un algoritmo, lo que la hace altamente eficiente; de otro modo no se podría transmitir. Este primer nivel de la actividad matemática no requiere la comprensión global del sistema.

El nivel tiene una gran riqueza matemática. Agrupa a la actividad propia de un curso de matemáticas para ingenieros, que consiste en trazar gráficas de curvas o en hacer cálculos 
de cinemática, resolver integrales de una sola variable, dobles y triples, la variable compleja y el álgebra para ingenieros, las series de Fourier y los primeros métodos para resolver ecuaciones diferenciales en derivadas parciales, las herramientas matemáticas de la Física para ingenieros y muchas otras cosas. Incluso, en opinión de Changeux y Connes (ob.cit.), la comprobación de la validez de un razonamiento en un Teorema corresponde al primer nivel, por lo que es de esperar que las computadoras pronto sean capaces de ello. Así que la matemática en la educación politécnica corresponde al primer nivel de la actividad matemática, caracterizado por la simple aplicación de algoritmos y donde la creatividad no es una necesidad apremiante, porque ejecutar un algoritmo sólo requiere seguir una serie de pasos previamente conocidos, es decir es una rutina. No hay el cuestionamiento ni la necesidad de modificar esa rutina.

\section{El segundo nivel}

El segundo nivel se define como aquel en el cual es posible elegir una estrategia para un fin determinado, como la resolución de un problema; y cambiar según sea el resultado. Un ejemplo de Changeux y Connes (ob.cit.) es el de alguien que de tanto hacer multiplicaciones hallara un método más sencillo de obtener el resultado. Esto supondría comprobar el método, comprender su finalidad y su mecanismo. Un computador aún es incapaz de hacer esto. Estar en este nivel implica que al cometer un error es posible hacer comparaciones con otros cálculos, lo que supone la comprensión del algoritmo utilizado. El estudiante se siente más involucrado en el problema porque "el segundo nivel empieza cuando los cálculos efectuados interactúan con un problema personal.” (Changeux y Connes, ob.cit; p. 87).

Los sentimientos juegan un papel esencial para pasar del primer al segundo nivel, así como la capacidad de crear jerarquías de valores, de utilizarlas y de modificarlas. Como ejemplo personal de construir jerarquías de valores, diremos que el Análisis Vectorial, que corresponde al Cálculo III, siempre nos ha parecido un tema más interesante que muchos otros porque abre las puertas a la comprensión física y matemática de la Teoría Electromagnética y la Mecánica de los Fluidos. Se le debería dedicar más tiempo del previsto en los programas. En este mismo orden de ideas, estar en este nivel supone que al equivocarnos surge un sentimiento de frustración o cuando la estrategia no es optima, se busca mejorarla. Experimentar el placer de haber encontrado un método más eficaz y rápido o 
angustiarse por no hallarlo, son también sentimientos que impulsan el paso al segundo nivel, así como la facultad de apreciar la calidad o el valor de un resultado o un Teorema.

Para pasar al segundo nivel es necesario entonces haber formalizado las operaciones utilizadas, haberlas jerarquizado en función del fin al que se adapta la estrategia elegida y participar realmente en lo que se hace. Una diferencia importante entre este nivel y el siguiente, el tercero, es que aquí nunca se pierde el hilo del pensamiento; nunca hay una distancia entre el funcionamiento del cerebro y el objeto al cual se aplica, porque la mente se concentra, durante cierto tiempo, en cumplir con cada uno de los pasos del algoritmo apropiado al problema en cuestión, a objeto de resolverlo, mientras que en el tercer nivel los investigadores suelen dejar de lado momentaneamente los esfuerzos para resolver un problema complejo que le ha consumido todos sus recursos, con el fin de propiciar el pensamiento lateral y la intuición del inconciente.

La definición de creatividad asociada al segundo nivel se corresponde con las ideas propuestas por Csikszentmihalyi (1998). Para este autor, el contexto socio-cultural es crucial para decidir si una idea o producto puede considerarse un acto creativo. Las bases de su definición son: una cultura que contenga reglas simbólicas, una persona que aporta novedad al campo simbólico y un ámbito de expertos que reconocen y validan la innovación. Creemos que estas ideas se pueden adaptar al entorno de un salón de clases.

Creemos que la asignación de pequeñas investigaciones matemáticas, tipo proyecto, permiten la transición del primer al segundo nivel. Como experiencia personal, fue planteado un problema en Matemáticas Especiales a un curso de 35 estudiantes del cuarto semestre de ingeniería UNEXPO-Barquisimeto. No tenía carácter obligatorio pero quien lo resolviese sería recompensado. Se les pidió que no acudieran a profesores para ayuda porque era una especie de experimento creativo, y pudimos apreciar algunos de los aspectos que estamos señalando. La pequeña investigación era demostrar la propiedad asociativa de la convolución de tres funciones $\mathrm{f}, \mathrm{g}$, y h. Para ello no existe una receta previa, a menos que quien lo haga sea un profesor experimentado. En el ejercicio deben jugar con la definición de convolución de funciones, tener cuidado con las notaciones y símbolos empleados, cambiar el orden de la integración en las integrales dobles, graficar regiones de integración y tener una estrategia para ir ensamblando todos estos conocimientos que van desde el Calculo I al IV, para lograr el fin propuesto. Aquí no se esta inventando nada, pero es que el segundo nivel, estrictamente 
hablando, no es el de la creación pero se inicia el avance hacia ella al prescindir más de la receta y construir una estrategia para lograr un fin.

\section{El tercer nivel}

El tercer nivel es el de la creación matemática tal como la han expuesto Poincaré, Hadamard, Changeux y Connes, Penrose y otros. Es el de la invención matemática propiamente dicha, que tiene como su núcleo las fases de incubación e iluminación. Una de sus propiedades básicas es la pérdida del hilo del pensamiento, que busca relajar el enfoque y permite el pensamiento lateral; lo que es impropio del segundo nivel. Puede definirse del siguiente modo: “el 'espíritu’, o el 'pensamiento’, está ocupado en una labor diferente, mientras que, de manera interna, podríamos decir subconsciente, se esta resolviendo el problema. Lo esencial es precisamente esta disociación entre el pensamiento presente, activo, y un funcionamiento del cerebro que no es aparente...” (Changeux y Connes, ob.cit.; p. 118).

En este nivel, las manifestaciones de creatividad pueden ocurrir en dos vías: resolver un problema ya planteado y que ha resistido el ataque de otros matemáticos o, aprovechando un problema ya planteado o una reflexión, crear herramientas de pensamiento que no existían previamente y que permiten develar una parte inexplorada de la matemática. La iluminación, propiedad distintiva del nivel, permite un cambio global de estrategia; creándose así un nuevo marco de pensamiento, para la aplicación de una nueva táctica. Cuando leemos sobre la invención matemática en los textos clásicos, se están refiriendo principalmente al tercer nivel, al que consideramos sumamente exigente y poco práctico para estudiantes de un curso normal de matemáticas para ingeniería por las razones antes expuestas.

\section{REFLEXIÓN FINAL}

A manera de conclusión, y utilizando una metáfora, diremos que la actividad matemática es un paisaje constituido por tres regiones: la primera es la de un saber instrumental y utilitario, donde reinan los algoritmos y las recetas para solucionar problemas, en consecuencia esta excluida la creatividad. Los planes de estudio de matemáticas para las escuelas de ingeniería se corresponden con este primer nivel. La tercera región es la de la creatividad e innovación dura, la de los matemáticos profesionales que dedican un tiempo 
prolongado a varios problemas simultáneamente, en un ambiente de investigación propicio y que deliberadamente estimulan las fases de incubación e iluminacion del modelo clásico de creatividad matemática, mediante una de las propiedades distintivas del nivel que la discontinuidad del hilo del pensamiento sobre la actividad matemática que se esta llevando a cabo, en aras de favorecer el pensamiento lateral y la iluminación. No hay límites de tiempo para el surgimiento del resultado creativo en este nivel.

La región intermedia entre las dos anteriores es la que nos interesa desde el punto de vista didáctico. Es esta una región difusa en donde ocurren los primeros indicios creativos sin llegar al descubrimiento notable, sino más bien modesto pero altamente motivador para el aprendizaje y la formación científica. El estudiante pasa de la mera aplicación de algoritmos, recetas y rutinas preestablecidas a cuestionar su eficacia, modificarlas y poder elegir entre varias estrategias para solucionar su problema. Por lo tanto, se siente más comprometido emocional y cognoscitivamente con su aprendizaje activo y con la solución de su problema matemático, dedicándole mayor tiempo, todo lo cual lo induce a asumir una actitud creativa. Allí debemos concentrar la atención los educadores en matemáticas.

En el ensayo hemos expuesto que, atendiendo a las limitaciones de tiempo, espacio y perfil profesional de los estudiantes de una universidad politécnica, la creatividad se puede fomentar proponiendo y ejecutando estrategias didácticas matemáticas que busquen rebasar el primer nivel, caracterizado por la aplicación de algoritmos y cálculos. Las estrategias pueden consistir en pequeñas investigaciones, a modo de proyectos, que involucren la aplicación de anteriores conocimientos tanto matemáticos como de otras ciencias, sea física, biología, ecología, etc., donde el estudiante se involucre más en el problema, construya jerarquías de valores, las utilice y modifique; experimente placer al encontrar un método más eficaz, aprecie el valor y la calidad de un resultado y no pierda el hilo de su pensamiento mientras este resolviendo su investigación, aparte de que la limitante tiempo no se lo permitiría. Así mismo, se angustie o sienta frustrado cuando su pequeña investigación no avanza como debiera. Estas actitudes configuran el marco general del segundo nivel, que no es el de la creatividad dura de los matemáticos insignes pero que puede crear las condiciones para que, en algunos casos muy reducidos y casi por iniciativa propia, se acceda al tercer nivel.

\section{REFERENCIAS}


Changeux, J y Connes, A. (1993). Materia de Reflexión. España. Tusquets Editores. Colección Metatemas.

Csikszentmihalyi, M. (1998). Creatividad. El Fluir y la Psicología del Descubrimiento y la Invención. España. Ediciones Paidós Ibérica, S. A.

Davis, P. y Hersh, R. (1998). The Mathematical Experience. Boston, New York. A Mariner Book. Houghton Mifflin Company

Hadamard, J. (1996). The Mathematician`s Mind. The Psychology of Invention in the Mathematical Field. Princeton, New Jersey. Princeton University Press.

Penrose, R. (1996). La Mente Nueva del Emperador. México. Consejo Nacional de Ciencia y Tecnología, Fondo de Cultura Económica.

Poincaré, H. (1963). Ciencia y Método. Madrid, España. Editorial Espasa-Calpe, S.A. Colección Austral.

Sriraman, B. (2004). The Characteristics of Mathematical Creativity. [Artículo en línea]. The Mathematics Educator. 14(1), 19-34 Disponible: http://www.umt.edu/math/reports/sriraman/Sriraman 7 2009.pdf. Archivo PDF[Consulta: 2011, Febrero 05] 